\title{
Variations on the Dirac string
}

\author{
Brad Cownden" \\ Department of Physics \& Astronomy, University of Manitoba, Winnipeg, Manitoba R3T 2N2, Canada \\ Andrew R. Frey ${ }^{\dagger}$ \\ Department of Physics, University of Winnipeg, 515 Portage Avenue, \\ Winnipeg, Manitoba R3B 2E9, Canada \\ and Department of Physics \& Astronomy, University of Manitoba, \\ Winnipeg, Manitoba R3T 2N2, Canada
}

(Received 1 August 2018; published 27 November 2018)

\begin{abstract}
Dirac's original solution of the nontrivial Bianchi identity for magnetic monopoles [1], which redefines the field strength along the Dirac string, diagonalizes the gauge and monopole degrees of freedom. We provide a variant of the Dirac string, which we motivate through a formal expansion of the Bianchi identity. We show how to use our variant prescription to study monopole electrodynamics without reference to a dual potential and provide some applications.
\end{abstract}

DOI: $10.1103 /$ PhysRevD.98.105013

\section{INTRODUCTION}

Magnetic monopoles, while experimentally elusive [2], hold a special place in particle physics; they complete electric-magnetic duality, provide a reason for charge quantization, and arise in the spontaneous breaking of many grand unification models. At the same time, there is a long tradition of reformulating the description of the monopole's interaction with the electromagnetic field since Dirac's original work [1,3]:

(i) Monopoles arise as solitons of broken gauge theories; the microscopic description as a semiclassical field configuration describes the monopole's interactions but requires tracking the full degrees of freedom (d.o.f.) of that gauge theory. While of course necessary for processes such as monopole creation and annihilation (see e.g., [4]), it may be computationally excessive when an effective description is valid.

(ii) In the absence of electric charges, the field strength can be defined in terms of a dual potential $\star F \equiv d \tilde{A}$, which couples to monopoles in the same way the vector potential couples to charges. Of course, if the fields of both charges and monopoles are of interest, the field strengths from both the potential and the

\footnotetext{
*cowndenb@myumanitoba.ca

†.frey@uwinnipeg.ca
}

Published by the American Physical Society under the terms of the Creative Commons Attribution 4.0 International license. Further distribution of this work must maintain attribution to the author(s) and the published article's title, journal citation, and DOI. Funded by SCOAP ${ }^{3}$. dual potential must be superposed in a "democratic" formalism (which makes it ill-suited to the quantum mechanics of charges and monopoles). This type of approach has been advocated at least since [5].

(iii) As pointed out originally in [6,7], the vector potential is not a globally defined function but a section of a fiber bundle. When the field strength has a nontrivial Bianchi identity, the potential is defined in at least two coordinate patches; the potentials in the two patches are related by a gauge transformation in the overlap of the two patches. While mathematically rigorous, treating the potential in this manner mixes the gauge and monopole d.o.f. because the overlap region moves with the monopole. This formalism also obscures the coupling between the potential and monopole (e.g., [7] switches to a dual potential formalism to derive the monopole's classical equation of motion, while [8] following [9] combined Dirac's formalism below with the rigorous gauge patching procedure).

(iv) The potential in a single coordinate patch extends to all of spacetime except for a half-line singularity extending from the monopole (Dirac's famous string singularity). In [1], Dirac noted that the electromagnetic field strength can be written in terms of a globally defined potential and an extra term supported on the string singularity. This approach separates the electromagnetic and monopole d.o.f. and elucidates the monopole-field strength coupling. However, it suffers some conceptual difficulties, including a singular field strength along the (arbitrarily chosen) string and a constraint that electric charges cannot intersect the Dirac string world 
sheet. Pragmatically, the semi-infinite string can be awkward.

In this paper, we present a new hybridization of Dirac's string formalism with the rigorous gauge patching prescription by allowing the Dirac string to end on an unphysical reference monopole, and we review the derivation of Dirac's string prescription from the dual potential formalism with an emphasis on the origin of the string. We also advocate for a particular configuration for the string and give a simple physical picture for its origin. The fixed prescription for the string means that the string world sheet embedding coordinates depend on the monopole position along the entire world sheet, unlike Dirac's original formalism; our approach presents computational advantages in several circumstances. The extension of our results to higher-dimensional monopolelike branes appears in the companion paper [10] by one of us. Throughout this paper, we work in four-dimensional Minkowski spacetime for simplicity. We list our conventions, particularly signs, in the Appendix.

\section{DIRAC'S STRING VARIABLES}

The Maxwell equations and Bianchi identities with magnetic sources are

$$
d \star F=-\star j, \quad d F=-\star \tilde{\jmath},
$$

where $j^{\mu}$ is the electric current and $\tilde{j}^{\mu}$ is the magnetic current. For a single monopole, the current is

$\tilde{J}^{\mu}=g \int_{\mathcal{M}} d \tau \partial_{\tau} X^{\mu}(\tau) \delta^{4}(x-X)=g u^{\mu}(t) \delta^{3}(\vec{x}-\vec{X})$,

where $g$ is the monopole charge, $\mathcal{M}$ is the monopole's worldline, $\tau$ is the worldline time, and $X^{\mu}$ is the monopole position. The second equality follows in a fixed reference frame with a worldline static gauge $\tau=t ; u^{\mu}$ is the monopole's 4-velocity.

Dirac's key observation stems from the fact that any conserved current (in Minkowski spacetime) can be written as the divergence of a two-form, or $\star \tilde{j}=d \star H$ for some $H$. In fact, the field of an isolated monopole provides such an $H$ subject to the additional constraint of the sourceless Maxwell equations. Instead, following Dirac [1], we define

$$
G^{\mu \nu}(x) \equiv \int_{\mathcal{N}} \bar{d} Y^{\mu} \wedge \bar{d} Y^{\nu} \delta^{4}(x-Y)
$$

where $\mathcal{N}$ is the world sheet of a string with boundary $\partial \mathcal{N}=\mathcal{M}-\mathcal{M}_{*}\left(\mathcal{M}_{*}\right.$ is a so-far arbitrary worldline), $Y^{\mu}(\tau, \sigma)$ is the string position at world sheet coordinates $\tau, \sigma$ with $\sigma$ increasing from $\mathcal{M}_{*}$ to $\mathcal{M}$, and $\bar{d}=d \tau \partial_{\tau}+d \sigma \partial_{\sigma}$ is the world sheet exterior derivative. This has divergence

$$
\begin{aligned}
(\star d \star G)^{\mu} & =\int_{\mathcal{N}} \bar{d} Y^{\nu} \wedge \bar{d} Y^{\mu} \partial_{\nu} \delta^{4}(x-Y) \\
& =-\int_{\mathcal{N}} \bar{d}\left[\delta^{4}(x-Y) \bar{d} Y^{\mu}\right] \\
& =(1 / g) \tilde{J}^{\mu}-(1 / g) \tilde{J}_{*}^{\mu}
\end{aligned}
$$

in terms of the currents of monopoles along $\mathcal{M}$ and $\mathcal{M}_{*}{ }^{1}$ As a result, the field strength defined as $F=d A-g \star G$ automatically solves the Bianchi identity for the dynamical monopole along $\mathcal{M}$ as long as $A$ includes the potential for a monopole with reference world line $\mathcal{M}_{*}$ (with appropriate gauge patching).

It is worth pausing now to discuss the configuration of the string. In Dirac's formalism, the string is a dynamical object with no kinetic term (in modern parlance, a tensionless string), so the world sheet $\mathcal{N}$ is completely arbitrary except for the specification of its boundary. ${ }^{2}$ Dirac furthermore locates the worldline $\mathcal{M}_{*}$ at spatial infinity, so the string becomes semi-infinite. However, we are free to specify the configuration of the string; a simple choice in a given reference frame is to choose the reference monopole to be stationary $\left(\mathcal{M}_{*}\right.$ at constant position $\left.\vec{X}_{*}\right)$ and the string to be the straight line from $\vec{X}_{*}$ to the monopole position $\vec{X}(t)$ at each time. We can build an arbitrary world sheet in this way by then extending a Dirac string from $\mathcal{M}_{*}$ to another reference worldline $\mathcal{M}_{* *}$, and so on, and then letting the reference worldlines approach each other. On the other hand, with a single line segment, the world sheet embedding coordinates $Y^{\mu}$ depend on the monopole position at all world sheet points $\tau, \sigma$ unlike in Dirac's formalism. We motivate this prescription for the Dirac string configuration in Sec. IV.

It is also instructive to consider the Dirac quantization of charge for different formalisms. With distinct gauge patches, quantization enforces the requirement that a single-value wave function in one coordinate patch remains single valued after the gauge transformation in passing to another patch. In Dirac's original formalism, there is only a single gauge, but the wave function picks up a phase under motion of the string equal to $g$ times the electric flux through the surface swept out by the world sheet-the surface is noncontractible since the charge cannot intersect the world sheet, as we see below. Since the surface swept out by the string is closed (including the point at infinity), charge quantization is given by the condition that the wave function remain single valued if we sweep the string around the charge. When the reference monopole is at a finite

\footnotetext{
${ }^{1}$ An additional sign enters at the last equality from the world sheet orientation as described in the Appendix.

${ }^{2}$ This arbitrariness reflects how a gauge transformation modifies where the potential of a monopole becomes singular (or more precisely the patching required for the potential), so the string can never develop a tension, even quantum mechanically, for an unbroken gauge symmetry.
} 
position, both mechanisms for charge quantization are possible. In particular, if the string world sheet is arbitrary but $\mathcal{M}_{*}$ is fixed, we can sweep the string in a closed surface around a charge, as per Dirac. With a set prescription for the world sheet as discussed above, however, the world sheet moves only if we move the reference position $\vec{X}_{*}$, so the string sweeps out open surfaces, removing the quantization condition. On the other hand, the potential must be defined in patches around the reference point, so single-valuedness of the wave function still leads to charge quantization.

The Dirac string formalism also provides a direct means of finding the monopole equation of motion, which is otherwise carried out indirectly through the dual potential formalism. Including the string coupling $G$ in the field strength, the variation of the Maxwell action with respect to the monopole and world sheet positions is

$$
\begin{aligned}
\delta S_{\mathrm{Max}}= & \frac{g}{4} \epsilon_{\mu \nu \lambda \rho} \int d^{4} x F^{\mu \nu}(x) \delta G^{\lambda \rho}(x) \\
= & \frac{g}{4} \epsilon_{\mu \nu \lambda \rho} \int d^{4} x \int_{\mathcal{N}} F^{\mu \nu}(x)\left\{\bar{d} Y^{\lambda} \wedge \bar{d} Y^{\rho} \delta Y^{\alpha} \partial_{Y^{\alpha}} \delta^{4}(x-Y)\right. \\
& \left.+\left[\bar{d} \delta Y^{\lambda} \wedge \bar{d} Y^{\rho}+\bar{d} Y^{\lambda} \wedge \bar{d} \delta Y^{\rho}\right] \delta^{4}(x-Y)\right\}
\end{aligned}
$$

We can now restrict to a world sheet integral, first converting the derivative on the delta function to one with respect to $x^{\alpha}$ and integrating by parts. Then, with all partial derivatives now with respect to $Y$,

$$
\begin{aligned}
\delta S_{\mathrm{Max}}= & \frac{g}{2} \epsilon_{\mu \nu \lambda \rho} \int_{\mathcal{N}}\left\{\bar{d}\left[F^{\mu \nu}(Y) \delta Y^{\lambda} \bar{d} Y^{\rho}\right]\right. \\
& +\frac{1}{2} \partial_{\alpha} F^{\mu \nu}(Y)\left(\bar{d} Y^{\alpha} \wedge \bar{d} Y^{\lambda} \delta Y^{\rho}\right. \\
& \left.\left.+\bar{d} Y^{\lambda} \wedge \bar{d} Y^{\rho} \delta Y^{\alpha}+\bar{d} Y \rho \wedge \bar{d} Y^{\alpha} \delta Y^{\lambda}\right)\right\} .
\end{aligned}
$$

The latter lines of (6) reorganize to

$$
\frac{g}{2} \int_{\mathcal{N}}(d \star F)_{\nu \lambda \rho} \bar{d} Y^{\nu} \wedge \bar{d} Y^{\lambda} \delta Y^{\rho}
$$

which would yield an interaction between the (unphysical) string - and therefore the monopole-with the electric current when the gauge fields are on shell. To avoid this unphysical result, Dirac imposed the additional condition that charges not intersect the string. The first term, on the other hand, gives an integral over $\mathcal{M}$ (assuming $\mathcal{M}_{*}$ fixed), ${ }^{3}$ so it combines with the variation of the monopole's kinetic term to give the magnetic Lorentz force equation

$$
\partial_{\tau} p_{\mu}=-g(\star F)_{\mu \nu} \partial_{\tau} X^{\nu}
$$

\footnotetext{
${ }^{3}$ With sign determined per footnote 1 .
}

Meanwhile, the electric charge couples to the redefined potential $A$, so its equation of motion turns out as usual except for contact terms with the Dirac string, which are forbidden due to Dirac's condition.

It is important to note that our derivation of the monopole equation of motion treated the potential $A$ as an independent d.o.f. in contrast to the case without a Dirac string and with gauge patches. In that case, there is no coupling between the monopole and gauge field, only a hidden dependence of $A$ on $X^{\mu}$ which should be treated as an explicit dependence. The Dirac string removes this explicit dependence from the vector potential, but $A$ does still have a "hidden" explicit dependence on the arbitrary reference position. The equation of motion for the reference position is $F^{\mu \nu} \delta F_{\mu \nu} / \delta X_{*}^{\lambda}=0$, which enforces the condition that the field strength is independent of the reference position. This condition determines the explicit dependence of the potential on $X_{*}^{\mu}$.

\section{THE STRING FROM THE DUAL POTENTIAL}

The dual potential formalism is useful in many applications since it translates the electrodynamics of monopoles into the more familiar electrodynamics of charges (and can even allow for the interaction of charges and monopoles since electromagnetism is linear). Here we review a derivation of the Dirac string from the dual potential, simplified from versions presented in $[11,12]$. In particular, we start with the dual field strength and potential and a magnetic current in the absence of electric charges (which lead to a Dirac string for the dual field strength) in order to emphasize that the Poincare duality itself leads to the Dirac string for the field strength in the presence of monopoles.

In form notation, the action for the dual electromagnetism with a single monopole is

$$
S=\int\left(-\frac{1}{2} \tilde{F} \wedge \star \tilde{F}+\tilde{A} \wedge \star \tilde{J}\right)
$$

where $\tilde{A}, \tilde{F}$ are the dual potential and field strength and $\tilde{J}$ is the monopole current (2). To dualize back to the "usual" potential in the absence of monopoles, we treat $\tilde{F}$ as the independent variable and add a Lagrange multiplier term $A d \tilde{F}$ to enforce the Bianchi identity for $\tilde{F}$ since $\tilde{A}$ does not appear. Solving the equation of motion for $\tilde{F}$ and substituting gives the usual Maxwell action.

With the monopole current, we must first find a way to eliminate the dual potential from (9). We proceed by recalling that any conserved current can be written as the divergence of a two-form (in other words, any coclosed form in Minkowski spacetime is coexact). In fact, the Maxwell equation $d \star \tilde{F}=\star \tilde{j}$ shows that $\tilde{j}$ can be written in terms of the monopole's field strength (which also satisfies $d \tilde{F}=0$ ). We have also seen above that the 
Dirac string coupling $G$ is another such two-form up to the subtraction of a current along the reference worldline $\mathcal{M}_{*}$, so we can write

$$
\begin{aligned}
S & =\int\left[-\frac{1}{2} \tilde{F} \wedge \star \tilde{F}+\tilde{A} \wedge d \star\left(g G+\tilde{F}_{*}\right)\right] \\
& =\int\left[-\frac{1}{2} \tilde{F} \wedge \star \tilde{F}+\tilde{F} \wedge \star\left(g G+\tilde{F}_{*}\right)-A^{\prime} \wedge d \tilde{F}\right],
\end{aligned}
$$

where $\tilde{F}_{*}$ is the dual field strength of the reference monopole and we have added the Lagrange multiplier term. This last term can be rewritten as $\tilde{F} d A^{\prime}$ up to a total derivative, so the equation of motion is $\star \tilde{F}=\star\left(g G+\tilde{F}_{*}\right)-d A^{\prime}$. The action is therefore classically equivalent to

$$
S=-\int \frac{1}{2}\left(d A^{\prime}-\star \tilde{F}_{*}-g \star G\right) \wedge \star\left(d A^{\prime}-\star \tilde{F}_{*}-g \star G\right)
$$

We recognize $-\star \tilde{F}_{*}=F_{*}$ as the reference monopole's field strength, so we define $d A^{\prime}+F_{*}=d A$ in terms of a potential $A$ with the appropriate gauge patches for the reference monopole. We are left with $F=d A-g \star G$ and the usual Maxwell action $-\int F \star F / 2$ including the Dirac string.

\section{AN INTERPRETATION OF THE STRING}

We can gain new insight into the Dirac string through the Bianchi identity. For clarity, we pick a reference frame and work in static gauge for the monopole (i.e., worldline time $\left.\tau=Y^{0}\right)$. In nonrelativistic notation, the Bianchi identity is

$$
\vec{\nabla} \cdot \vec{B}=g \delta^{3}(\vec{x}-\vec{X}), \quad \vec{\nabla} \times \vec{E}+\partial_{t} \vec{B}=-g \partial_{t} \vec{X} \delta^{3}(\vec{x}-\vec{X}) .
$$

Our goal is to diagonalize the d.o.f., removing the explicit dependence of $A_{\mu}$ on the monopole position. We carry out a formal expansion of the monopole current around a static reference monopole at fixed position $\vec{X}_{*}$; therefore, we end up with a static gauge patching procedure around $\vec{X}_{*}$ and will see that the remaining terms can be organized as a contribution to the field strength. ${ }^{4}$

We begin by writing

$$
\begin{aligned}
\delta^{3}(\vec{x}-\vec{X})= & \delta^{3}\left(\vec{x}-\vec{X}_{*}\right) \\
& +\sum_{n=1}^{\infty} \frac{1}{n !} \delta X^{i_{1}} \cdots \delta X^{i_{n}} \nabla_{i_{1}}^{*} \cdots \nabla_{i_{n}}^{*} \delta^{3}\left(\vec{x}-\vec{X}_{*}\right),
\end{aligned}
$$

\footnotetext{
${ }^{4}$ This is the approach taken in [13] for D3-branes.
}

where $\vec{\nabla}^{*}$ is the gradient with respect to $\vec{X}_{*}$ and $\delta \vec{X}=\vec{X}-\vec{X}_{*}$. Converting one of the derivatives to one with respect to $\vec{x}$, the terms in the sum can be written as a divergence, so defining

$$
\begin{aligned}
\vec{B}= & \vec{\nabla} \times \vec{A} \\
& -g \delta \vec{X} \sum_{n=0}^{\infty} \frac{1}{(n+1) !}(\delta \vec{X})^{n, i_{1} \cdots i_{n}}\left(\vec{\nabla}^{*}\right)_{i_{1} \cdots i_{n}}^{n} \delta^{3}\left(\vec{x}-\vec{X}_{*}\right),
\end{aligned}
$$

where $\vec{A}$ includes the potential of a fixed monopole at $\vec{X}_{*}$ and solves the scalar equation of (12). Subtracting this contribution from the vector part of the Bianchi identity similarly extracts a curl from the expansion of the delta function. We can therefore define

$$
\begin{aligned}
\vec{E}= & -\vec{\nabla} \Phi-\partial_{t} \vec{A}+g\left(\partial_{t} \delta \vec{X} \times \delta \vec{X}\right) \\
& \times \sum_{n=0}^{\infty} \frac{n+1}{(n+2) !}(\delta \vec{X})^{n, i_{1} \cdots i_{n}}\left(\vec{\nabla}^{*}\right)_{i_{1} \cdots i_{n}}^{n} \delta^{3}\left(\vec{x}-\vec{X}_{*}\right)
\end{aligned}
$$

to solve the Bianchi identity.

To confirm that this approach separates the gauge and monopole d.o.f., we can vary the Maxwell action with respect to $\vec{X}$ and, with some manipulation, find the magnetic Lorentz force equation by treating the potentials as independent variables. As with the Dirac string, the variation of the action also includes terms proportional to the sourceless Maxwell equations and derivatives of $\delta^{3}\left(\vec{x}-\vec{X}_{*}\right)$.

As an alternate approach, we can consider the Dirac string with $\mathcal{M}_{*}$ a static worldline at $\vec{X}_{*}$. Then, as we suggested in the section above, take $\mathcal{N}$ at any fixed time to be the line segment from $\vec{X}_{*}$ to $\vec{X}$. Then we can choose a static gauge ${ }^{5} t=Y^{0}(\tau, \sigma)=\tau$ and $\vec{Y}(\tau, \sigma)=\vec{X}_{*}+\sigma \delta \vec{X}(t)$ with $0 \leq \sigma \leq 1$. Then the Dirac string coupling becomes

$$
\begin{aligned}
G^{0 i} & =\int_{0}^{1} d \sigma \delta X^{i} \delta^{3}\left(\vec{x}-\vec{X}_{*}-\sigma \delta \vec{X}\right) \\
& =\delta X^{i} \sum_{n=0}^{\infty} \frac{1}{n !}(\delta \vec{X})^{n, i_{1} \cdots i_{n}}\left(\vec{\nabla}^{*}\right)_{i_{1} \cdots i_{n}}^{n} \delta^{3}\left(\vec{x}-\vec{X}_{*}\right) \int_{0}^{1} d \sigma \sigma^{n}
\end{aligned}
$$

and similarly

$$
\begin{aligned}
G^{i j}= & \left(\partial_{t} X^{i} \delta X^{j}-\partial_{t} X^{j} \delta X^{i}\right) \sum_{n=0}^{\infty} \frac{1}{n !}(\delta \vec{X})^{n, i_{1} \cdots i_{n}}\left(\vec{\nabla}^{*}\right)_{i_{1} \cdots i_{n}}^{n} \\
& \times \delta^{3}\left(\vec{x}-\vec{X}_{*}\right) \int_{0}^{1} d \sigma \sigma^{n+1}
\end{aligned}
$$

\footnotetext{
${ }^{5}$ The argument goes through with little change for any function of $\tau$.
} 
after expanding the delta function. Carrying out the integral and using the usual relation between the field strength $F_{\mu \nu}$ and fields $\vec{E}, \vec{B}$, we find (14) and (15).

So the particular solution of the Bianchi identity that we found by expanding the delta function around $\vec{X}_{*}$ is a particular realization of the Dirac string. As a result, we see that the expansion of the delta function separates the explicit dependence of the field strength on the monopole position from the potential. This also tells us that the Dirac string is a way of treating a monopole's motion as a fluctuation (even a large one) around a fixed position. The key difference with Dirac's arbitrary string is that this interpretation suggests treating the embedding coordinates of the string as dependent on the monopole position along the entire world sheet. In fact, we have done this explicitly in deriving (16) and (17).

For future reference, it is useful to give the exact expressions for the fields with the linear string configuration:

$$
\begin{aligned}
\vec{E} & =-\vec{\nabla} \Phi-\partial_{t} \vec{A}+g\left(\partial_{t} \vec{X} \times \delta \vec{X}\right) \int_{0}^{1} d \sigma \sigma \delta^{3}\left(\vec{x}-\vec{X}_{*}-\sigma \delta \vec{X}\right), \\
\vec{B} & =\vec{\nabla} \times \vec{A}-g \delta \vec{X} \int_{0}^{1} d \sigma \delta^{3}\left(\vec{x}-\vec{X}_{*}-\sigma \delta \vec{X}\right) .
\end{aligned}
$$

The displacement $\vec{D}$ and field $\vec{H}$ are determined as usual from $\vec{E}, \vec{B}$ in linear media, including the Dirac string contribution.

\section{THE STRING AS A SOURCE}

The Dirac string in the field strength automatically solves the Bianchi identity, so the Bianchi identity no longer determines the field strength associated with the dynamic monopole. Instead, as Dirac realized, the string coupling $G$ leads to an effective electric current as a source for $d A$, the field strength away from the Dirac string. In particular, $d \star d A=-g d G$, so the effective current is $j_{\text {eff }}=g \star d G$.

For practical use, we again choose a line segment configuration as in Sec. IV. The effective charge is

$$
\begin{aligned}
\rho_{\mathrm{eff}} & =j_{\mathrm{eff}}^{0} \\
& =-g\left(\partial_{t} \vec{X} \times \delta \vec{X}\right) \cdot \vec{\nabla}\left[\int_{0}^{1} d \sigma \sigma \delta^{3}\left(\vec{x}-\vec{X}_{*}-\sigma \delta \vec{X}\right)\right],
\end{aligned}
$$

where the gradient is with respect to $\vec{x}$. Evaluating the effective current in the static gauge is slightly subtler. We have

$$
\begin{aligned}
j_{\mathrm{eff}, i}= & -g \epsilon_{i 0 j k} \int d \tau \int_{0}^{1} d \sigma\left(\sigma \partial_{\tau} X^{j} \delta X^{k}\right) \\
& \times \partial_{t}\left[\delta(t-\tau) \delta^{3}\left(\vec{x}-\vec{X}_{*}-\sigma \delta \vec{X}\right)\right] \\
& +g \epsilon_{i j 0 k} \int_{0}^{1} d \sigma \delta X^{k} \partial_{j} \delta^{3}\left(\vec{x}-\vec{X}_{*}-\sigma \delta \vec{X}\right) .
\end{aligned}
$$

We must take care to convert the $t$ derivative to a $\tau$ derivative and integrate by parts where the delta function in time is differentiated. We are left with

$$
\begin{aligned}
\vec{J}_{\mathrm{eff}}= & g \partial_{t}\left[\left(\partial_{t} \vec{X} \times \delta \vec{X}\right) \int_{0}^{1} d \sigma \sigma \delta^{3}\left(\vec{x}-\vec{X}_{*}-\sigma \delta \vec{X}\right)\right] \\
& -g \delta \vec{X} \times \vec{\nabla}\left[\int_{0}^{1} d \sigma \delta^{3}\left(\vec{x}-\vec{X}_{*}-\sigma \delta \vec{X}\right)\right] .
\end{aligned}
$$

This is worth two comments. First, the current for arbitrary linear motion of the monopole, with $\vec{X}_{*}$ chosen to lie on the line, is

$$
\vec{J}_{\mathrm{eff}}=-g \delta \vec{X} \times \vec{\nabla}\left[\int_{0}^{1} d \sigma \delta^{3}\left(\vec{x}-\vec{X}_{*}-\sigma \delta \vec{X}\right)\right] .
$$

Taking $\delta \vec{X}$ to lie along the $\pm z$ axis, Eq. (22) is the surface current in the $\pm \phi$ direction of an infinitely tightly wound, infinitesimally thin solenoid stretching from $\vec{X}_{*}$ to $\vec{X}$. The direction and magnitude of the current are precisely such that the flux into the solenoid at $\vec{X}_{*}$ (which is spherically symmetric since the solenoid is thin) precisely cancels the magnetic flux due to the reference monopole at $\vec{X}_{*}$. Similarly, the flux out of the solenoid at $\vec{X}$ precisely reproduces the flux of the dynamical monopole. Second, the current is conserved, as it must be, if we allow for singular distributions. The solenoidal current (22) is clearly conserved, and the other terms take the form $\rho_{\text {eff }}=-\vec{\nabla} \cdot \vec{k}, \vec{\jmath}_{\text {eff }}=\partial_{t} \vec{k}$, which is manifestly conserved.

In media, the effective charge and current follow from $\vec{\nabla} \cdot \vec{D}$ and $\vec{\nabla} \times \vec{H}$, so derivatives of the permittivity and permeability will appear in general. For piecewise constant dielectric constant and permeability, it is natural to define the effective charge and current in a piecewise manner as well.

Specifying a string configuration that depends explicitly on the monopole position along the world sheet gives a well-defined effective current from the string. In principle, we can now solve for the electromagnetic fields for arbitrary monopole motion as a superposition of the magnetic field from the fixed reference monopole and the effective current. The linear string configuration seems particularly well suited to this type of calculation.

\section{APPLICATIONS}

As we have noted previously, there are technical difficulties in the theory of electrodynamics with monopoles. Here we present several possible applications in which the linear Dirac string configuration yields simplifications.

We indicated above that one such application is a direct determination of radiation from moving monopoles, including energy loss in dielectric materials (such as 
Cherenkov radiation), ${ }^{6}$ which may be useful for monopole search experiments. While an infinite, arbitrarily moving Dirac string is unwieldy, the linear Dirac string configuration gives a well-defined current, which is simply a growing or shrinking solenoid in many contexts. In the presence of materials, as noted, it is necessary to write the fields nonrelativistically as $\vec{E}, \vec{B}$ and include the permittivity and permeability in defining $\vec{D}, \vec{H}$.

We have emphasized that the Dirac string formulation separates the gauge and monopole d.o.f.; as a result, it provides a basis for Hamiltonian and therefore quantum treatments. In this form, the introduction of extra unphysical d.o.f. for the Dirac string leads to constraints [16]. On the other hand, when the Dirac string takes the linear configuration, the entire string depends on the monopole position. In the variation of the action, these appear through terms proportional to the sourceless Maxwell equations [see Eq. (7)] and are trivial on shell. On the other hand, these terms can contribute off shell, for example in the path integral. Understanding how these contribute to the quantum mechanics of monopoles is an interesting question. Meanwhile, the analogous terms for D3-branes (see the discussion below) also play an important role in the four-dimensional effective action of type IIB string theory $[10,13]$.

The Dirac string formalism straightforwardly extends to curved spacetimes and higher dimensions, and the linear configuration for the string becomes a world sheet with geodesics as constant-time slices. A second end point to the string on $\mathcal{M}_{*}$ therefore allows us to use the Dirac string for monopoles on compact manifolds. While the magnetic Gauss law constraint (net magnetic charge on a compact manifold vanishes) means that any Dirac string can end on oppositely charged physical monopoles, an arbitrary reference end point allows for a cleaner separation of the dynamics of the different monopoles. Furthermore, it allows us to avoid having multiple Dirac strings end on one monopole in the case that the monopoles on a compact manifold carry different numbers of magnetic quanta (e.g., there are two monopoles of charge +1 and one of charge -2). Again, in the case of higher-dimensional monopolelike branes, monopole charge can dissolve into the flux of other fields, so the Dirac string from a monopole may not even have another monopole on which to end, necessitating the reference end point.

Finally, higher-dimensional branes of string theory are magnetic sources for various rank form fields. As in electromagnetism, a mathematically rigorous treatment considers the potentials as sections, while a Dirac-like formalism allows the separation of the gauge and brane d.o.f. (see [13]; one of us will detail this formalism in the forthcoming [10]). The formalism therefore provides an alternative to the democratic (dual potential) formalism

\footnotetext{
${ }^{6}$ See $[14,15]$ for the energy loss rate in different approximations.
}

for the derivation of brane equations of motion. It is particularly helpful for a careful accounting of d.o.f., as needed in dimensional reduction. Determining the lowerdimensional effective action is also an off-shell calculation, and extra terms from the generalized Dirac string world volume (which vanish on shell) are critical to account for all the kinetic terms required by supergravity $[10,13]$.

\section{ACKNOWLEDGMENTS}

A. R. F. thanks K. Dasgupta, N. Afshordi, and L. Boyle for interesting discussions. B. C. and A. R. F. are supported by the Natural Sciences and Engineering Research Council of Canada Discovery Grant program. Part of this work was supported by the Perimeter Institute for Theoretical Physics. Research at Perimeter Institute is supported by the Government of Canada through the Department of Innovation, Science and Economic Development and by the Province of Ontario through the Ministry of Research and Innovation.

\section{APPENDIX: CONVENTIONS}

Here we briefly lay out our conventions, including signs. To start, we take the mostly plus metric convention with $\epsilon_{0123}=+1$. The Hodge star for a differential $p$ form is given by $(\star F)_{\mu_{1} \cdots \mu_{4-p}}=(1 / p !) \epsilon_{\mu_{1} \cdots \mu_{4-p}} \nu_{1} \cdots \nu_{p} F_{\nu_{1} \cdots \nu_{p}}$, so $\star \star F=$ $(-1)^{p(4-p)+1}$.

With standard conventions (see $[14,17]$ ), the Maxwell equations with magnetic currents included are

$$
\begin{array}{ll}
\vec{\nabla} \cdot \vec{E}=\rho, & \vec{\nabla} \times \vec{B}-\partial_{t} \vec{E}=\vec{\jmath}, \\
\vec{\nabla} \cdot \vec{B}=\tilde{\rho}, & \vec{\nabla} \times \vec{E}+\partial_{t} \vec{B}=-\overrightarrow{\tilde{\jmath}},
\end{array}
$$

where $\rho, \vec{j}$ are the electric charge and current and $\tilde{\rho}, \overrightarrow{\tilde{j}}$ are the magnetic. In relativistic notation, we take $A^{\mu}=(\Phi, \vec{A})$, $j^{\mu}=(\rho, \vec{J})$ (and likewise for the magnetic current), so $F_{0 i}=-E_{i}, F_{i j}=\epsilon_{i j k} B_{k}$. The Maxwell equations become

$\partial_{\mu} F^{\mu \nu}=-j^{\nu}, \quad \partial_{\mu} F_{\nu \lambda}+\partial_{\nu} F_{\lambda \mu}+\partial_{\lambda} F_{\mu \nu}=-\epsilon_{\mu \nu \lambda \rho} \tilde{J}^{\rho}$,

or $d \star F=-\star j$ and $d F=-\star \tilde{j}$ in terms of forms. The dual field strength $\tilde{F} \equiv \star F$ therefore satisfies the dual Maxwell equations $d \tilde{F}=-\star j$ and $d \star \tilde{F}=+\star \tilde{j}$. As a result, the dual electric current is usually defined as $-\tilde{\jmath}$; for simplicity of comparison, we do not introduce this sign.

Finally, we define the Dirac string coupling $G$ as a form integral over the string world sheet coordinates $\tau, \sigma$. We choose the orientation by taking integration measure $d^{2} \sigma=d \tau \wedge d \sigma=-d \sigma \wedge d \tau$. 
[1] P. A. M. Dirac, The theory of magnetic poles, Phys. Rev. 74, 817 (1948).

[2] B. Acharya et al. (MoEDAL Collaboration), Search for magnetic monopoles with the MoEDAL forward trapping detector in $2.11 \mathrm{fb}^{-1}$ of $13 \mathrm{TeV}$ proton-proton collisions at the LHC, Phys. Lett. B 782, 510 (2018).

[3] P. A. M. Dirac, Quantized singularities in the electromagnetic field, Proc. R. Soc. A 133, 60 (1931).

[4] T. Vachaspati, Creation of Magnetic Monopoles in Classical Scattering, Phys. Rev. Lett. 117, 181601 (2016).

[5] F. Rohrlich, Classical theory of magnetic monopoles, Phys. Rev. 150, 1104 (1966).

[6] T. T. Wu and C. N. Yang, Dirac monopole without strings: Monopole harmonics, Nucl. Phys. B107, 365 (1976).

[7] T. T. Wu and C. N. Yang, Dirac's monopole without strings: Classical Lagrangian theory, Phys. Rev. D 14, 437 (1976).

[8] K. Hirata, Classical Lagrangian theory of Dirac's monopole: Avoiding Dirac's veto, Phys. Lett. 81B, 169 (1979).

[9] R. A. Brandt and J. R. Primack, Avoiding Dirac's veto in monopole theory, Phys. Rev. D 15, 1798 (1977).
[10] A. R. Frey, Dirac branes for Dirichlet branes (to be published).

[11] S. Deser, A. Gomberoff, M. Henneaux, and C. Teitelboim, P-brane dyons and electric magnetic duality, Nucl. Phys. B520, 179 (1998).

[12] K. Lechner and P. A. Marchetti, Duality invariant quantum field theories of charges and monopoles, Nucl. Phys. B569, 529 (2000).

[13] B. Cownden, A. R. Frey, M. C. David Marsh, and B. Underwood, Dimensional reduction for D3-brane moduli, J. High Energy Phys. 12 (2016) 139.

[14] J. D. Jackson, Classical Electrodynamics, 2nd ed. (Wiley, New York, 1975); 3rd ed. (Wiley, New York, 1998).

[15] J. Gea-Banacloche, K. E. Cahill, and D. Rossbach, Energy loss by slow magnetic monopoles, Lett. Nuovo Cimento 37, 145 (1983).

[16] A. P. Balachandran, R. Ramachandran, J. Schechter, K. C. Wali, and H. Rupertsberger, Hamiltonian formulation of monopole theories with strings, Phys. Rev. D 13, 354 (1976).

[17] D. J. Griffiths, Introduction to Electrodynamics, 4th ed. (Pearson, London, 2012). 